\title{
Errar no sólo es humano
}

To err is not only human

Norma Cipatli Ayuzo-del Valle*

Han pasado ya 20 años desde que el Institute of Medicine (IOM) en Estados Unidos publicó el famoso artículo "To err is human», citado en más de 20,000 artículos médicos; en el cual se describió, entre otras cosas, que los errores en la medicación alcanzaban el séptimo lugar como causa de muerte, además de los costos sanitarios derivados de estos errores humanos. Este artículo tuvo un gran impacto, e impulsó a autoridades y agentes implicados en la asistencia sanitaria a abordar la seguridad del paciente como un objetivo prioritario.

Se define «error» como una equivocación hecha en el proceso del cuidado de un paciente que provoca o tiene el potencial de causar daño. ${ }^{2}$ El error médico puede ser el incumplimiento de un plan terapéutico, el establecimiento de un plan erróneo, un error de prescripción, de omisión, casi omisión o cuasi-falla (si no alcanzó a llegar al paciente). La mayoría de los errores médicos no son causados de forma arbitraria, sino que están relacionados con factores y equivocaciones a nivel de la organización. ${ }^{3}$

El mismo IOM relaciona problemas como la mala caligrafía de las prescripciones médicas y el mal manejo de los expedientes, y recomienda medidas como el uso de tecnología para reducir los errores de medicación. ${ }^{4}$ Con dichas medidas se pensaría que los errores médicos disminuirían de forma importante, planteándose la meta de reducir su incidencia hasta en 50\% en los siguientes cinco años ${ }^{5}$ a partir de la publicación. Con el avance de la tecnología en los últimos años, se han implementado expedientes electrónicos, recetas electrónicas, gestores de citas, dispensación y administración de medicamentos por código de barras y hasta softwares de simulación para mejora de gestiones hospitalarias. ${ }^{6}$

Bajo el pensamiento de que la introducción de la tecnología sería indispensable para la mejora de la calidad de atención y de esa forma evitar los errores humanos, esta implementación no sólo falló en disminuir la tasa de errores, sino que, incluso, generó un aumento de los mismos de 98,000 en 1999 a 190,000 en el 2004.7

JAMA reporta para el 2005 que la introducción de la informática paradójicamente incrementó el riesgo de errores que llegan al paciente. ${ }^{8}$

Recientemente (2019), un artículo español9 describió cómo la implementación de la tecnología informática no mejora la seguridad del proceso de prescripción, empeorando incluso el apartado de frecuencia de administración (63 vs 57\%).

Otro ejemplo es el fallo que ocurrió en la agenda electrónica de programación de mamografías en Inglaterra, reportado en el 2018, que excluyó de la agenda a pacientes con sospecha de lesiones, ${ }^{10}$ que pudo haber ocasionado retrasos diagnósticos y hasta una cantidad importante de muertes sin ser error humano.

Estudios de bombas de infusión han descrito también errores en la configuración de las mismas, que llevan a equivocaciones importantes en la administración
* Pediatra del Grupo Ángeles. Académica por la Academia Mexicana de Pediatría y la Academy for Eating Disorders.

Correspondencia: NCAV, cipatlimd@yahoo.com Conflicto de intereses: La autora declara que no tiene.

Citar como: Ayuzo-del Valle NC. Errar no sólo es humano. Rev CONAMED 2020; 25(2): 103-104. doi: 10.35366/94395

Financiamiento: Ninguno.

Recibido: 20/01/2020. Aceptado: 31/03/2020 
de medicamentos derivados de los aparatos tecnológicos."

La introducción de medios electrónicos ha traído consigo otros errores distintos al humano, relacionados con la tecnología de los que aún se desconoce su impacto total.

Conforme aumenta exponencialmente la diversidad de uso de estos gadgets, también incrementa el riesgo de daño para los pacientes derivados de un mal uso, fallos de integración, errores de programación, caídas del sistema o malfuncionamiento de software y hardware. ${ }^{12}$

Sin embargo, el reporte de los errores de la tecnología aún no se encuentra clasificado para su análisis, ni hay indicadores de medición de los mismos. Es necesaria la creación de incidentes de seguridad que permita la clasificación de estos daños y así poder medir de forma objetiva el errar de tecnologías que afectan directamente la salud del paciente y reestructurar ante nuevos tiempos que Errar no es sólo humano.

\section{AGRADECIMIENTO}

A la Dra. Brenda Ayuzo de la Universidad Autónoma de Guadalajara.

\section{BiBLIOGRAFÍA}

1. Kohn L, Corrigan J, Donaldson M. To err is human. Builiding a safer health system. Washington: National Academy Press; 1999 .
2. Domínguez G, Espinoza L. Errores en medicina. Ciruj Gral. 2010; 1 (32): 100-102.

3. Leape L. Making health care safe: Are we up to it? J Ped Surg. 2004; 39: 258-266.

4. Aspen P, Wolcott J, Bootman JL et al. The National Academies Press; Committee on identifying and preventing medication error Institute of medicine of the national academies. Preventing medication error 2006.

5. Bogner SM. Revisiting to err is human a decade later. Biomedical Instrumentation and Technology. 2009; 43 (6): 476-479

6. Ayuzo del Valle Cipatli, Villafuerte CJC. Uso de software de simulación para mejorar los tiempos de atención en el departamento de emergencias. Salud Pública de México. 2018; 60 (3): 373-374, ISSN 1606-7916.

7. Healthcare. Patient Safety in American Hospitals. Healtcare 2004. Available in: www.healthgrades.com/media/english/ pdf/hg_patient_study_final.pdf.

8. Koppel R, Metlay JP, Cohen A, Abaluck B, Localio AR, Kimmel SE, Strom BL. Role of computerized physician order entry systems in facilitating medical errors. JAMA. 2005; 293 (10): 1197-1203.

9. Jiménez-Muñoz AB, Martínez-Mondejar B, Saiz-Ladera GM, Romero-Ayuso DN, Paniagua-Alcaraz C, Criado-Álvarez JJ. Puede la informatización evitar errores de medicación? Análisis de los errores potenciales de prescripciones al alta. OFIL. 2019; 29 (3): 201-209.

10. Senthilingam M. Up to 270 women may have died after England breast cancer screeninf failures. CNN 2018. Avalable in: https://edition.cnn.com/2018/05/02/health/ uk-breast-cancer-screening-scandal-int//index.html.

11. Horsky J, Zhang, Patel. To err is not entirely human: complex technology and user cognition. J Biomed Inform. 2005; 38 (4): 264-266.

12. Monje B, Tovar P, Campos S, Tutau G. Errar ya no es solo humano. OFIL. 2019; 29 (2): 164. 